\title{
Consistent Multi-view Reconstruction from Epipolar Geometries with Outliers
}

\author{
Daniel Martinec and Tomáš Pajdla* \\ Center for Machine Perception, Department of Cybernetics \\ Faculty of Elec. Eng., Czech Technical University in Prague \\ Karlovo nám. 13, 12135 Praha, Czech Republic \\ \{martid1,pajdla\}@cmp.felk.cvut.cz
}

\begin{abstract}
This paper presents a method for automatic 3D-reconstruction from a set of images. The main contribution of this paper is a robust integration of the partial correspondences from image pairs provided by an existing correspondence estimator into a reconstruction consistent with all images of the scene. Projective shape and motion is estimated by factorization of a matrix containing the images of all scene points. Outlier detection is based on RANSAC paradigm and the trifocal tensors. Compared to previous methods, this method can handle perspective views, occlusions, and outliers in image correspondences jointly. The main novelty of this paper is the fusion of a correspondence estimator [9] for wide base-line stereo and the method for outlier detection [7]. It appears that the method is able to detect outliers that cannot be detected using the epipolar geometry alone and therefore it is suitable for integrating with wide base-line stereo from image pairs. The new method is demonstrated by experiments with laboratory and outdoor image sets and some results on metric reconstruction are shown.
\end{abstract}

\section{Introduction}

RANSAC on correspondences from image pairs usually exploits the epipolar geometry constraint, which says that an image point must lie on the corresponding epipolar line. However, there still may be wrong correspondences left among those satisfying epipolar geometry. Such wrong correspondences, called outliers, cannot be detected using the epipolar geometry alone. Moreover, it can happen

\footnotetext{
* Jiří Matas and Ondřej Chum from the Czech Technical University in Prague provided the image pair matches, Tomáš Werner from the University of Oxford provided the routine for computing camera matrices from six points across three images and the bundle adjustment, Andrew Zisserman from the University of Oxford kindly provided the Valbonne data, and K. Mikolajczyk and C. Schmid from INRIA Rhone-Alpes, France, provided the House data. This research was supported by the following grants: GACR 102/01/0971, GACR 102/03/0440, BeNoGo IST-2001-39184, Aktion 34p24, CTU 8306713, Net CEEPUS SK-042, MSMT Kontakt 22-2003-04, and MSM 212300013.
} 
in some degenerate cases, e.g. when the overlapping area of the two images is almost planar, that the estimated epipolar geometry is completely wrong and there is no way to find it out using the epipolar geometry. Because of these two reasons, geometric constraints from more than two views have to be used sometimes.

Our motivation in this paper was to use multiview constraints for detecting outliers in such cases when the two-view geometry is not sufficient, see Fig. 1.
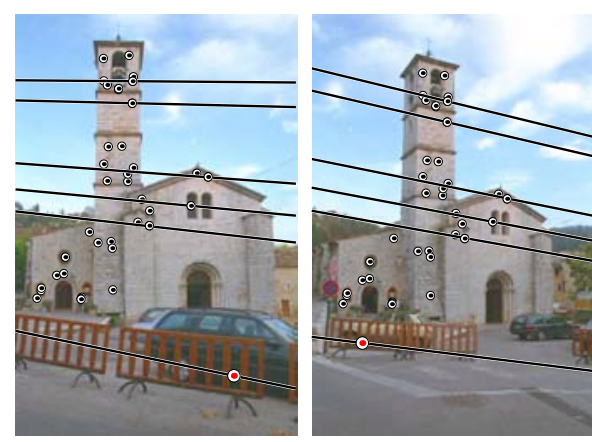

(a) An outlier satisfying epipolar geometry in an image pair. Only some epipolar lines are drawn
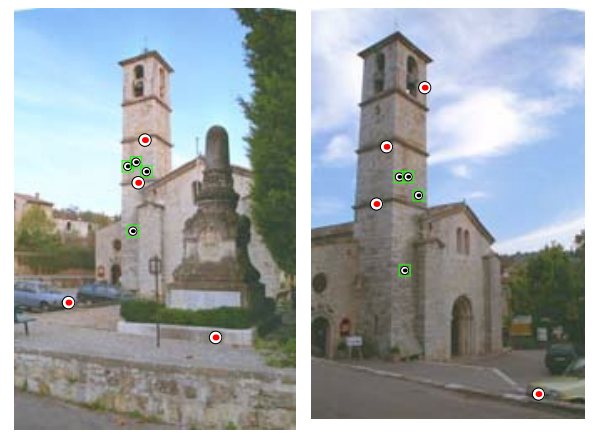

(b) Correspondences found using the multiview geometry when the epipolar geometry failed

Fig. 1. (a) Corresponding points satisfying the epipolar geometry may be outliers. (b) The multiview geometry can provide matches even when the epipolar geometry fails

Tomasi \& Kanade [12] developed a factorization method of the measurement matrix for scene reconstruction with an orthographic camera. Their method as well as Jacobs' method [6] can handle occlusions. Sturm and Triggs [11] extended this method from affine to perspective projections but without occlusions. Martinec \& Pajdla [8] solved reconstruction with both perspective projections and occlusions. Heyden [5] presented a reconstruction method from affine images with outliers but occlusions are not handled. Recently he extended the method into the perspective case [4]. We presented a method [7] for outlier detection so that reconstruction from perspective images is solved when occlusions and outliers are present jointly. The method is independent of image ordering and treats all data uniformly. No six-tuple [3] and not even a three-tuple [10] of points seen in all images is needed.

The contribution of this paper is a robust integration of the partial correspondences from image pairs provided by correspondence estimator [9] into a more consistent reconstruction from all images of the scene. An automatic 
method for 3D-reconstruction from plain images is achieved as the combination of the method [7] for outlier detection by factorization applied on the automatic correspondence estimator [9] and followed by projective reconstruction [8].

After problem formulation in Section 2, the way of obtaining correspondences will be explained in Section 3. Outlier detection and projective reconstruction will be briefly sketched in Sections 4 and 5, respectively. Experiments and conclusion come in Sections 6 and 7.

\section{Problem Formulation}

Suppose a set of $n 3 \mathrm{D}$ points is observed by $m$ perspective cameras. Not all points are visible in all views. There also may be outliers, i.e. mismatches in correspondences. The goal is to reject outliers and to recover $3 \mathrm{D}$ structure (point locations) and motion (camera locations) from the remaining image measurements that are called inliers.

Let $\mathbf{X}_{p}$ be the unknown homogeneous coordinate vectors of 3D points, $\mathrm{P}^{i}$ the unknown $3 \times 4$ projection matrices, and $\mathbf{x}_{p}^{i}$ the measured homogeneous coordinate vectors of image points, where $i=1, \ldots, m$ labels images and $p=1, \ldots, n$ labels points. Due to occlusions, $\mathbf{x}_{p}^{i}$ are unknown for some $i$ and $p$.

The basic image projection equation says that $\mathbf{x}_{p}^{i}$ are the projections of $\mathbf{X}_{p}$ up to unknown scale factors $\lambda_{p}^{i}$, which will be called (projective) depths:

$$
\lambda_{p}^{i} \mathbf{x}_{p}^{i}=\mathrm{P}^{i} \mathbf{X}_{p}
$$

The complete set of image projections can be gathered into a matrix equation:

$$
\left[\begin{array}{cccc}
\lambda_{1}^{1} \mathbf{x}_{1}^{1} & \lambda_{2}^{1} \mathbf{x}_{2}^{1} & \ldots & \lambda_{n}^{1} \mathbf{x}_{n}^{1} \\
\times & \lambda_{2}^{2} \mathbf{x}_{2}^{2} & \ldots & \times \\
\vdots & & \ddots & \vdots \\
\lambda_{1}^{m} \mathbf{x}_{1}^{m} & \times & \ldots & \lambda_{n}^{m} \mathbf{x}_{n}^{m}
\end{array}\right]=\underbrace{\left[\begin{array}{c}
\mathrm{P}^{1} \\
\vdots \\
\mathrm{P}^{m}
\end{array}\right]}_{\mathrm{P}} \underbrace{\left[\mathbf{X}_{1} \ldots \mathbf{X}_{n}\right]}_{\mathbf{X}}
$$

where marks $\times$ stand for unknown elements, which could not be measured due to occlusions, $\mathrm{X}$ and $\mathrm{P}$ stand for structure and motion, respectively. The $3 m \times n$ matrix $\left[\mathbf{x}_{p}^{i}\right]_{i=1 . . m, p=1 . . n}$ will be called the measurement matrix, shortly MM. MM may have (and in most cases does have) some missing elements and outliers.

\section{Obtaining Correspondences}

Correspondences across all images were established from matches between pairs of images obtained using the method by Matas et al. [9] exploiting a special kind of distinguished regions, so called extremal regions. Matches obtained using this method satisfy the epipolar constraint but are not guaranteed to be the true correspondences. Therefore, an outlier detection technique may still be needed to reject remaining outliers that can only be found using more than two images. 


\begin{tabular}{|l|l|}
\hline Scene Valbonne sequence (Oxford) & $\begin{array}{l}14 \text { images }[768 \times 512] \\
297 / 80.74 \%\end{array}$ \\
Correspondences / missing data & $\begin{array}{l}82.52 \% \text { of } \lambda_{p}^{2} \text { known } \\
\mathbf{5 0}(6.24 \% \text { of } 801 \text { image points) } \\
10 / 4 \\
20 \text { estimation: sequence }\end{array}$ \\
Outliers & $\begin{array}{l}233 / 24 / 64 \text { out of } 297 \\
\text { Rec. / not-reconstructed cameras } \\
\text { Rec. / partially rec. / not-rec. corresp / } 1.82 \text { pxl (from inliers) } \\
\text { Mean / maximal reprojection error }\end{array}$ \\
\hline
\end{tabular}

\begin{tabular}{|l|l|}
\hline Scene Valbonne all pairs (Oxford) & 14 images $[768 \times 512]$ \\
Correspondences / missing data & $376 / 73.27 \%$ \\
\hline$\lambda_{p}^{2}$ estimation: sequence & $51.53 \%$ of $\lambda_{p}^{2}$ known \\
Outliers & $\mathbf{3 9 6}(28.14 \%$ of 1407 image points) \\
Rec. / not-reconstructed cameras & $14 / 0$ \\
Rec. / partially rec. / not-rec. corresp. & $271 / 32 / 105$ out of 376 \\
Mean / maximal reprojection error & $\mathbf{0 . 4 5} / 3.66$ pxl (from inliers) \\
\hline
\end{tabular}

376 correspondences
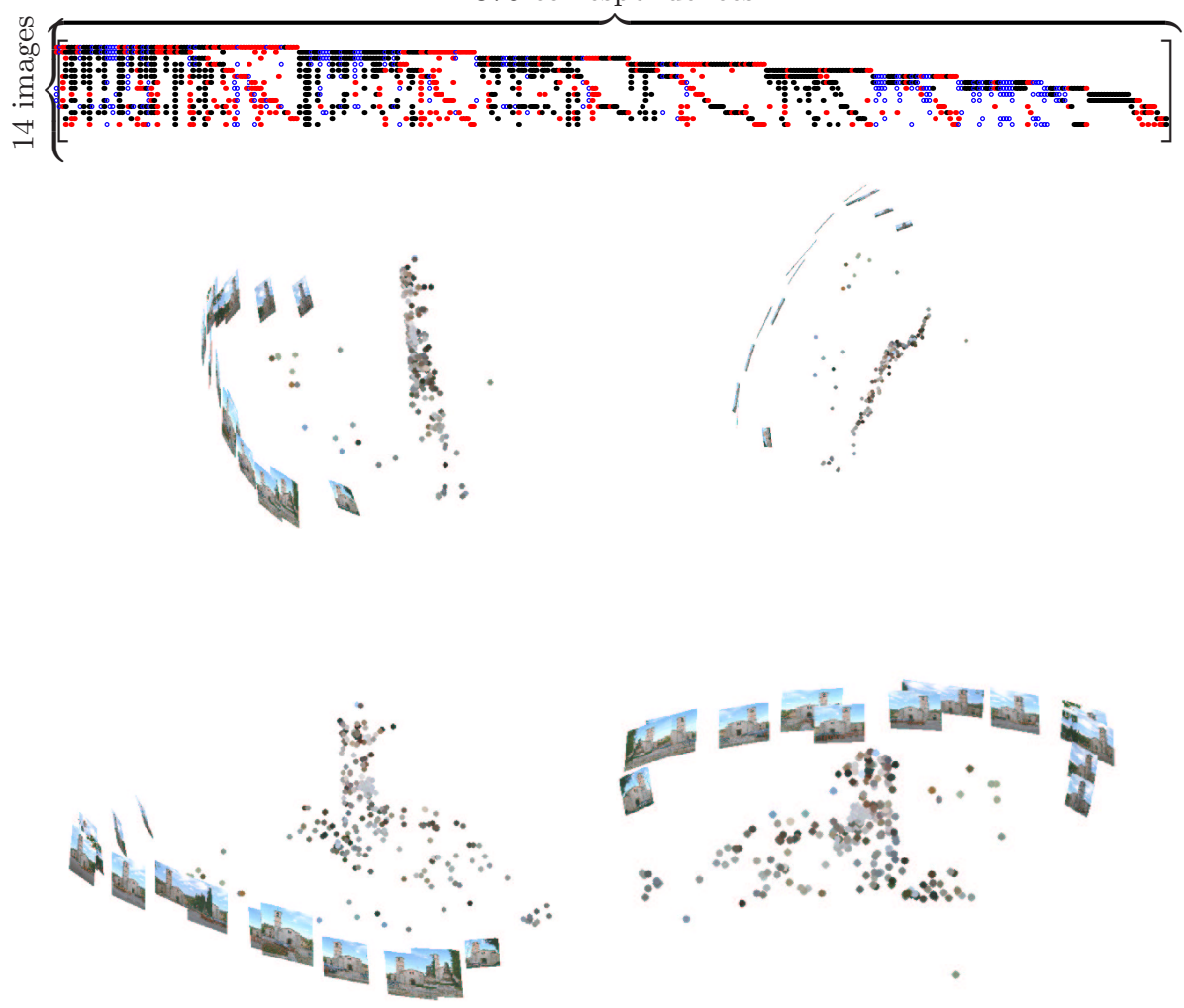

Fig. 2. The Valbonne scene reconstructed using sequence only (top) and using 'all image pairs' (lower). Note significantly larger amount of the reconstructed data for all image pairs: number of cameras as well as points is higher than for sequence only 
The transitivity assumption was adopted in the process of building the measurement matrix. Whenever there are pair-wise correspondences between images 1-2 and 2-3, it can be expected that there is a pair-wise correspondence between images $1-3$. The assumption holds for true correspondences, however, this is not always the case using method [9]. If some pair-wise matches are conflicting, i.e. the found match between images 1-3 differs from the match expected because of matches between images $1-2$ and $2-3$, it is not clear which pair-wise match is true and which is wrong. The task could be stated so that a maximal set of not conflicting pair-wise correspondences could be searched for. Such a problem leads to a greedy algorithm which is highly non-linear. We did not try to solve it in an optimal manner [1]. Thanks to the possibility of using the outlier detection method [7], conflicts among pair-wise correspondences could be simply ignored and false matches revealed in the subsequent outlier detection stage. Image pairs were read in a random order. The matches between image pairs were placed into the measurement matrix so that the overlapping matches were joined and the conflicting ones were ignored.

\section{Outlier Detection}

Method [7] was used for outlier detection. The method assumes that the amount of inliers is significantly larger than the amount of outliers. This is true thanks to matcher [9] that discards all matches that do not satisfy consistent epipolar geometries between image pairs. The main idea of [7] is that minimal configurations of points in triples of images are sufficient to validate inliers reliably. The RANSAC paradigm is used. Trifocal tensors are computed from randomly selected minimal 6-tuples of points in triples of images. After the tensor estimation, the number of points consistent with the tensor is counted. If there are sufficiently enough consistent points, those not used to estimate the trifocal tensor receive one positive vote. The voting is repeated until points in the measurement matrix are sufficiently sampled. The points that obtain zero or a very small number of votes are rejected as outliers. Inliers are used by the method described in [8] to obtain a projective reconstruction. The set of inliers can be further enlarged by an iterative process.

Method [7] can inspect correspondences among at least three images because the trifocal tensors are used. However, there may be some right correspondences between two images only. Each image point marked by method [7] as outlier is finally verified by sampling pair-wise correspondences in the corresponding column of MM. Each such sample is checked by reconstructing the 3D point using the known cameras. If the reprojection errors in both images are small, the correspondence (and the image point) is validated.

\section{Projective Reconstruction}

Method [8] was used for projective reconstruction because it can deal with occlusions in various configurations like in the data from wide base-line multiple 
view stereo. It is a method for recovery of projective shape and motion from multiple images by factorization of a matrix containing the images of all scene points (MM). Compared to previous methods, this method can handle perspective views and occlusions jointly. The projective depths of image points are estimated by the method of Sturm \& Triggs [11] using the epipolar geometry. Occlusions are solved by the extension of the method by Jacobs [6] for filling the missing data. This extension can exploit the geometry of the perspective camera so that both points with known and unknown projective depths are used. Many ways of combining the two methods exist, and the one with the best results was presented in [8].

\section{Experiments}

In all experiments, pair-wise matches were found using method [9]. The accuracy for outlier detection using method [7] was set to 5 pixels. For each experiment, one image, an error table, and the structure of MM are provided. The table includes the scene name, the number of images and their sizes, the number of found correspondences, the amount of missing data, the chosen strategy for depth estimation (see [8]), the amount of detected outliers, the number of reconstructed and not-reconstructed cameras, reconstructed, partially reconstructed, and notreconstructed correspondences, and the reprojection errors of the reconstruction without outliers. In structure of MM, "•" stands for outliers, "•" for image points with depths estimated using the fundamental matrices while "O" stands for image points with depths estimated in the subsequent process of filling MM, and " stand for the missing data.

The Valbonne scene was reconstructed in two different ways, see Fig. 2. First, $m-1$ image pairs were taken in a sequence and second, all $\left(\begin{array}{c}m \\ 2\end{array}\right)$ image pairs were used. As could be expected, the latter produced significantly larger amount of the reconstructed data: number of cameras as well as points was higher. Note that the sequence could be reconstructed using some sequential technique, e.g. [2], on the other hand, the data from all image pairs need to be treated by some robust method with missing data. Our factorization method [7] was well suited for the task. Moreover, in the Valbonne data in sequence, the number of correspondences among the four last images was too low to form a trifocal constraint; that is why these images could not be reconstructed by any sequential algorithm using the trifocal constraint (like [2]) at all.

Results of reconstructions of the Movi-house, the Shelf and the House scene are summarized in Fig. 3. Maximal errors in the Movi-house and the House scene are higher than 5 pixels set in the outlier detection accuracy. It is because of the following reason. The outlier detection accuracy is used for validation of the sampled three- and two-tuples of image points in columns of MM. There is no additional checking on the join of the validated $k$-tuples of image points. That is why the 3D-point reconstructed using more images can have larger reprojection error in some of them (although such 3D-point can be expected to be reconstructed more precisely). For more details, see [7]. 


\begin{tabular}{|l|l|}
\hline Scene Movi-house (CMP) & 14 images $[512 \times 512]$ \\
Correspondences / missing data & $101 / 49.28 \%$ \\
\hline$\lambda_{p}^{2}$ estimation: sequence & $44.47 \%$ of $\lambda_{p}^{2}$ known \\
Outliers & $\mathbf{2 0 7}(44.90 \%$ of 461 image points) \\
Rec. / not-reconstructed cameras & $9 / 0$ \\
Rec. / partially rec. / not-rec. corresp. & $67 / 33 / 34$ out of 101 \\
Mean / maximal reprojection error & $\mathbf{0 . 7 5} / 5.27$ pxl (from inliers) \\
\hline
\end{tabular}

101 correspondences

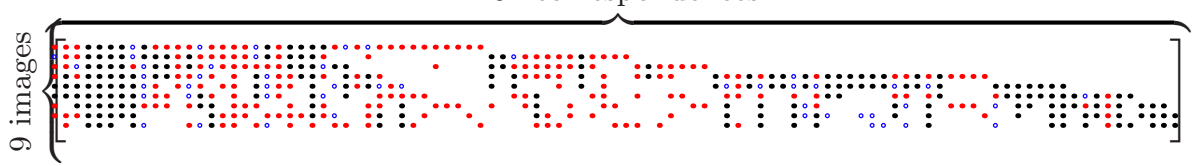

\begin{tabular}{|l|l|}
\hline Scene Shelf $(C M P)$ & 12 images $[1200 \times 1600]$ \\
Correspondences / missing data & $1953 / 72.64 \%$ \\
\hline$\lambda_{p}^{i}$ estimation: central image No. 4 & $75.00 \%$ of $\lambda_{p}^{i}$ known \\
Outliers & $\mathbf{4 1 4}(6.46 \%$ of 6411 image points) \\
Rec. / not-reconstructed cameras & $12 / 0$ \\
Rec. / partially rec. / not-rec. corresp. & $1839 / 72$ / 114 out of 1953 \\
Mean / maximal reprojection error & $\mathbf{0 . 5 1} / 4.90$ pxl (from inliers) \\
\hline
\end{tabular}
1953 correspondences

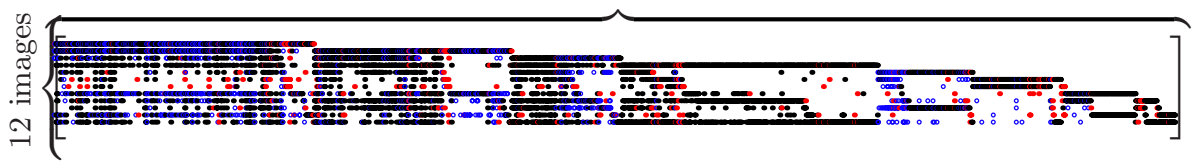

\begin{tabular}{|l|l|}
\hline Scene House $(C M P)$ & 14 images $[640 \times 800]$ \\
Correspondences / missing data & $1073 / 73.64 \%$ \\
\hline$\lambda_{p}^{2}$ estimation: central image No. 1 & $50.26 \%$ of $\lambda_{p}^{i}$ known \\
Outliers & $\mathbf{1 4 1 2}(38.40 \%$ of 3677 image points) \\
Rec. / not-reconstructed cameras & $13 / 0$ \\
Rec. / partially rec. / not-rec. corresp. & 787 / $175 / 286$ out of 1073 \\
Mean / maximal reprojection error & $\mathbf{0 . 5 1 ~ / ~ 8 . 3 1 ~ p x l ~ ( f r o m ~ i n l i e r s ) ~}$ \\
\hline
\end{tabular}

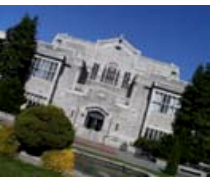

1073 correspondences

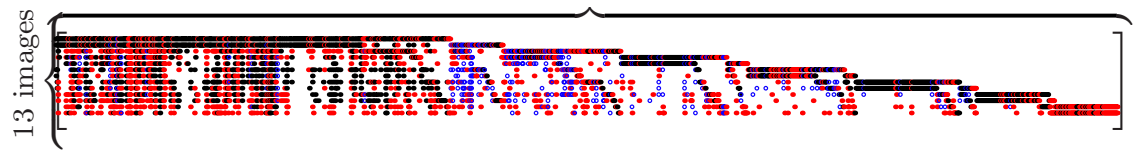

Fig. 3. Scenes reconstructed using all image pairs 


\subsection{Metric Reconstruction}

To demonstrate correctness of the reconstructions, an approximation of a metric reconstruction was computed. The linear method for auto-calibration using the absolute dual quadric [3] was applied. Lower part of Fig. 2 shows the metric reconstruction of the Valbonne scene using 'all image pairs'. Only this scene is shown because the linear algorithm for auto-calibration produced some reasonable output for this scene only. It is recommended in [3] to use the non-linear bundle adjustment on the output of the linear method.

\section{Conclusion}

A fully automatic method for 3D-reconstruction from plain images from perspective cameras was developed. It is the combination of the method [7] for outlier detection applied on the automatic correspondence estimator [9] and followed by projective reconstruction [8]. Tests on laboratory and outdoor scenes showed its applicability in wide base-line multiple view stereo while its applicability for sequences was presented in [7].

\section{References}

1. V. Ferrari, T. Tuytelaars, and L. Van Gool. Wide-baseline multiple-view correspondences. In Proceedings of the CVPR, 2003. In print.

2. A. W. Fitzgibbon and A. Zisserman. Automatic camera recovery for closed or open image sequences. In Proc. ECCV, volume I, pages 311-326. Springer-Verlag, June 1998.

3. R. Hartley and A. Zisserman. Multiple View Geometry in Computer Vision. Cambridge University Press, Cambridge, UK, 2000.

4. A. Heyden. Personal communication, 2002.

5. D. Q. Huynh and A. Heyden. Outlier detection in video sequences under affine projection. In Proc. IEEE Conf. on CVPR, pages 695-701, December 2001.

6. D. Jacobs. Linear fitting with missing data: Applications to structure from motion and to characterizing intensity images. In CVPR, pages 206-212, 1997.

7. D. Martinec and T. Pajdla. Outlier detection for factorization-based reconstruction from perspective images with occlusions. In Proceedings of the Photogrammetric Computer Vision, volume B, pages 161-164, 2002.

8. D. Martinec and T. Pajdla. Structure from many perspective images with occlusions. In Proceedings of the European Conference on Computer Vision, volume II, pages 355-369, Berlin, Germany, May 2002. Springer-Verlag.

9. J. Matas, O. Chum, M. Urban, and T. Pajdla. Robust wide baseline stereo from maximally stable extremal regions. In Proceedings of the British Machine Vision Conference, volume 1, pages 384-393, London, UK, September 2002. BMVA.

10. C. Rother and S. Carlsson. Linear multi view reconstruction with missing data. In Proceedings of the ECCV'02, volume II, pages 309-324, 2002.

11. P. Sturm and B. Triggs. A factorization based algorithm for multi-image projective structure and motion. In ECCV96(II), pages 709-720, 1996.

12. C. Tomasi and T. Kanade. Shape and motion from image streams under orthography: A factorization method. In $I J C V(9)$, No. 2, pages 137-154, November 1992. 